\title{
SOURCE: A Semi-automatic Tool for Spring Monitoring Data Analysis and Aquifer Characterization
}

\section{Stefano Lo Russo}

Politecnico di Torino

\section{Enrico Suozzi}

Politecnico di Torino

Martina Gizzi (D martina.gizzi@polito.it)

Politecnico di Torino https://orcid.org/0000-0001-7791-3260

\section{Glenda Taddia}

Politecnico di Torino

\section{Research Article}

Keywords: Mountain Springs, Hydrogeology, Hydrology, Python App, Spring Vulnerability

Posted Date: March 10th, 2021

DOI: https://doi.org/10.21203/rs.3.rs-227065/v1

License: (c) (i) This work is licensed under a Creative Commons Attribution 4.0 International License. Read Full License 

characterization

1. Department of Environment, Land and Infrastructure Engineering (DIATI), Politecnico di Torino, C.so Duca degli Abruzzi 24, 10129 Torino, Italy.

* Corresponding author: Martina Gizzi, martina.gizzi@ polito.it ORCID: 0000-0001-7791-3260

\section{Submitted to Environmental Earth Sciences}

Name of tool: SOURCE, a semi-automatic tool for Spring mOnitoring data analysis and aqUifeR

\section{Abstract}

It has become increasingly necessary to optimise mountain groundwater resource management and comprehend resource recharging systems from a hydrogeological perspective in order to formulate adequate resource protection strategies. Analysing mountain spring behaviour and aquifer characteristics can be time consuming, so new automated techniques and software tools are needed to estimate hydrogeological parameters and understand exhaustion dynamics of groundwater resources.

19 This paper introduces SOURCE, a new semi-automatic tool that automates the hydrogeological characterisation of water springs and provides proper estimations of the vulnerability index, as well as autocorrelation and cross-correlation statistical coefficients. SOURCE rapidly processed input data from the Mascognaz 1 spring (Aosta Valley) water probes and meteorological station to provide graphical outputs and values for the main hydrodynamic parameters.

24 Having a single software package that contains all the main methods of water spring analysis could potentially reduce analysis times from a few days to a few hours.

Keywords: Mountain Springs, Hydrogeology, Hydrology, Python App, Spring Vulnerability 


\section{Introduction}

28 Mountain aquifers represent one of the largest and most valuable sources of water in northern Italy and are necessary to meet the water needs of the population. In recent decades, different hydrological issues, such as the gradual drying up of many springs, low discharge rates during dry months, and formerly perennial springs

31 becoming seasonal, have been reported in studies from throughout the Italian Alps and Apennines (Cambi and Dragoni 2000; Fiorillo et al. 2007; Gattinoni and Francani 2010). Additionally, because mountain springs are fed by shallow groundwater resources, they are often highly vulnerable to contamination (Christie et al. 2013; Amanzio et al. 2015).

Optimizing the current and future management of mountain groundwater resources and understanding their recharging systems from a hydrogeological perspective is necessary for developing adequate resource management strategies. In addition, groundwater resources must be correctly quantified to provide information for the assessment of the effects of climate change on water resources (Middelkoop et al. 2001; Tague et al. 2012). In this context, new automated techniques and tools need to be applied to the estimation of aquifer hydrogeological parameters in order to fully understand the dynamics of exhausting available groundwater resources. based on the Boussinesq and Maillet equations (Boussinesq 1904; Maillet 1905). The Boussinesq equation is used to determine hydrogeological parameters, while the Maillet exponential formula generates good fits for hydrograph recession curves and accurately describes recession phenomena over long periods (Kovács et al. 2005); any deviation from the exponential trend may indicate the presence of hydraulic anisotropies (Amit et al. 2002; Fiorillo 2014). However, in some cases, the exponential method was found to overestimate the period of the influenced regime and underestimate the dynamic volume of the aquifer (Dewandel et al. 2003). Various recent studies have expanded autocorrelation and cross-correlation methods and applied them to mountain spring monitoring datasets. In particular, the univariate (autocorrelation) method has been used to analyse the characteristics and structure of individual time series; the bivariate (cross-correlation) method has been used to investigate the connection between input and output time series (Amanzio et al. 2015; Lo Russo et al. 2018). Several applications of auto- and cross-correlation methods examining the relation between 
rainfall and daily spring discharge are available in the literature on karst spring environments (Angelini 1997; Larocque et al. 1998; Panagopoulos and Lambrakis 2006).

Fiorillo and Doglioni (2010), Lo Russo et al. (2015) and Banzato et al. (2017) have recently demonstrated how aquifer drainage models and mountain spring vulnerability can be evaluated by analysing continuous measurements of discharge (Q), precipitation $(\mathrm{P})$, temperature $(\mathrm{T})$ and electrical conductivity (EC). Different methods have been proposed to assess the vulnerability level of an aquifer (Gogu and Dassargues 2000). As springs are usually located in mountainous zones, the use of parametric methods such as SINTACS (Civita and De Maio 1997), DRASTIC (Aller et al. 1987) or GOD (Foster 1987) is often impossible; these methods are primarily based on hydrostratigraphic information usually unavailable in mountainous areas since core drillings are somewhat rare in such locations. However, spring monitoring datasets continuously recorded by multiparametric probes are usually available; these data can be used to properly analyse groundwater vulnerability in mountain areas. Utilizing monitored parameters, Galleani et al. (2011) proposed a new analytical approach called the VESPA (Vulnerability Estimator for Spring Protection Areas) index, which is a useful methodological tool to assess the behaviour of mountain spring drainage systems through analysing spring responses to different recharge impulses.

Because the analytical examination of individual recession periods can generate inconsistencies related to the complexity of groundwater circulation, analysing the recorded values for mountain springs by comparing different recession periods and applying different methodologies is essential for properly characterizing recharge systems (Gizzi et al. 2020). To do this, data processing times must be shortened; researchers and applied hydrogeologists must develop new automated techniques and tools to apply in mountain aquifer analysis.

Different types of automated calculation codes have been developed to properly analyse spring hydrographs. The RC software developed by the Hydro Office of the Department of Hydrogeology of Comenius University in Bratislava (in collaboration with the Department of Hydrogeology and Geothermal Energy of the Geological Survey of Slovak Republic) and the USGS GW Toolbox (Barlow et al. 2017) are two well-known examples. However, these software do not allow for estimation of the vulnerability index using methods such as VESPA, and they do not apply autocorrelation and cross-correlation functions to analyse recorded signals. 
82 This paper introduces SOURCE (a semi-automatic tool for Spring mOnitoring data analysis and aqUifeR

83 CharactErization), a program that automates the hydrogeological characterisation of spring aquifers. Input data

84 (flow rate, temperature, hydraulic conductivity and rainfall) for particular time intervals can be selected and 85 uploaded in a formatted Excel file. The data can be processed, providing graphical outputs and values for the 86 main hydrodynamic parameters of the analysed aquifer.

87 The main functionalities of this tool are presented through a case study of the Mascognaz mountain springs 88 (Aosta Valley, north-western Italy).

89 The beta version of this software has been developed within the framework of the INTERREG ITALY90 SWITZERLAND RESERVAQUA project, which aims to quantify and identify water reserves in order to 91 protect cross-border mountain water springs like the Mascognaz springs.

\section{Methods}

\subsection{Case study: The Mascognaz springs}

The Mascognaz springs are one of the most important test sites in the Aosta Valley mountain sector. Over the last decade, several projects by researchers from Politecnico di Torino have installed sophisticated instruments, such as multiparametric water probes, different types of sensors and a meteorological station, to continuously monitor the two Mascognaz springs and collect information on their recharging systems. This equipment and the data it has continuously gathered are readily available to researchers, allowing them to accurately study how climate change influences aquifer recharge in a mountain basin not fed by a glacier. In addition, trend data for the Mascognaz springs can be compared to meteorological trend data for the valley. 


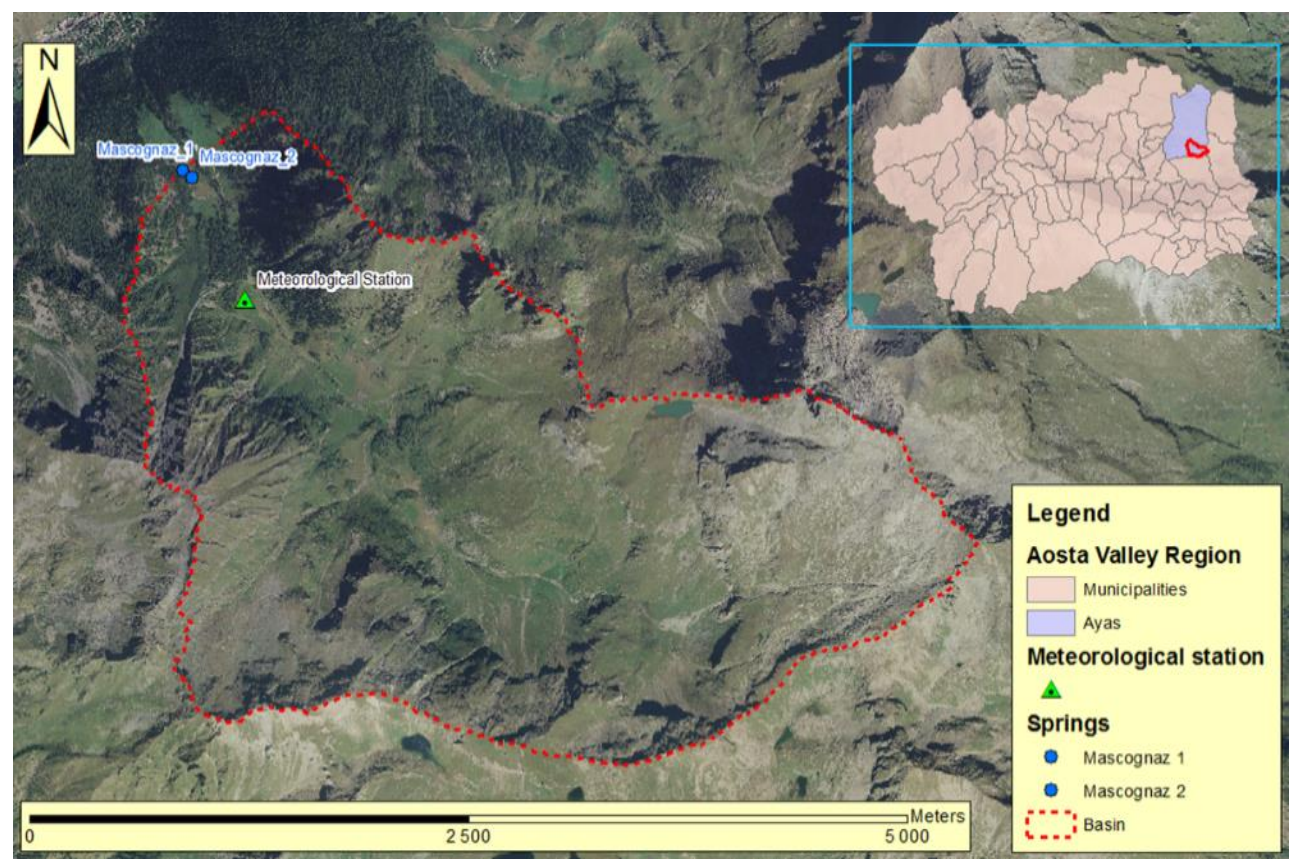

Figure 1: Mascognaz springs location

101 Both Mascognaz springs (Mascognaz 1 and Mascognaz 2) are located inside the Mascognaz Valley (Ayas

102 Municipality, Aosta Valley), at an elevation of $1870 \mathrm{~m}$ above sea level (Fig. 1). The Mascognaz Valley has a 103 typical alpine climate, with cold winters and cool summers. Autumn has the highest monthly rainfall, with 104 approximately $110 \mathrm{~mm}$ per month. Summer has the lowest monthly rainfall, with a mean of about $30 \mathrm{~mm}$ per 105 month.

106 The Mascognaz springs are located within the Combin geological complex, which belongs to the Piedmont

107 Zone geological sequence and consists of metabasalts and subordinate Mesozoic metasediments representative 108 of the local impermeable bedrock (Dal Piaz 1992). Overlaying Quaternary cover sheets consist mostly of 109 glacial and landslide deposits of variable thickness. A highly permeable shallow aquifer supplies the 110 Mascognaz springs. The Quaternary deposits over the Mascognaz springs catchment area $\left(10 \mathrm{~km}^{2}\right)$ have a 111 maximum estimated depth of $20 \mathrm{~m}$.

112 Due to the suitable monitoring datasets provided by the multiparameter probes and meteorological station, the 113 Mascognaz springs were selected as an ideal case study for this study (Fig. 2). Extensive information on the 114 local hydrogeological setting and the catchment area, which indicated that the groundwater divide and 
115 watershed boundaries coincide, is useful for properly understanding and characterizing the results obtained

116 from the water-spring analysis.

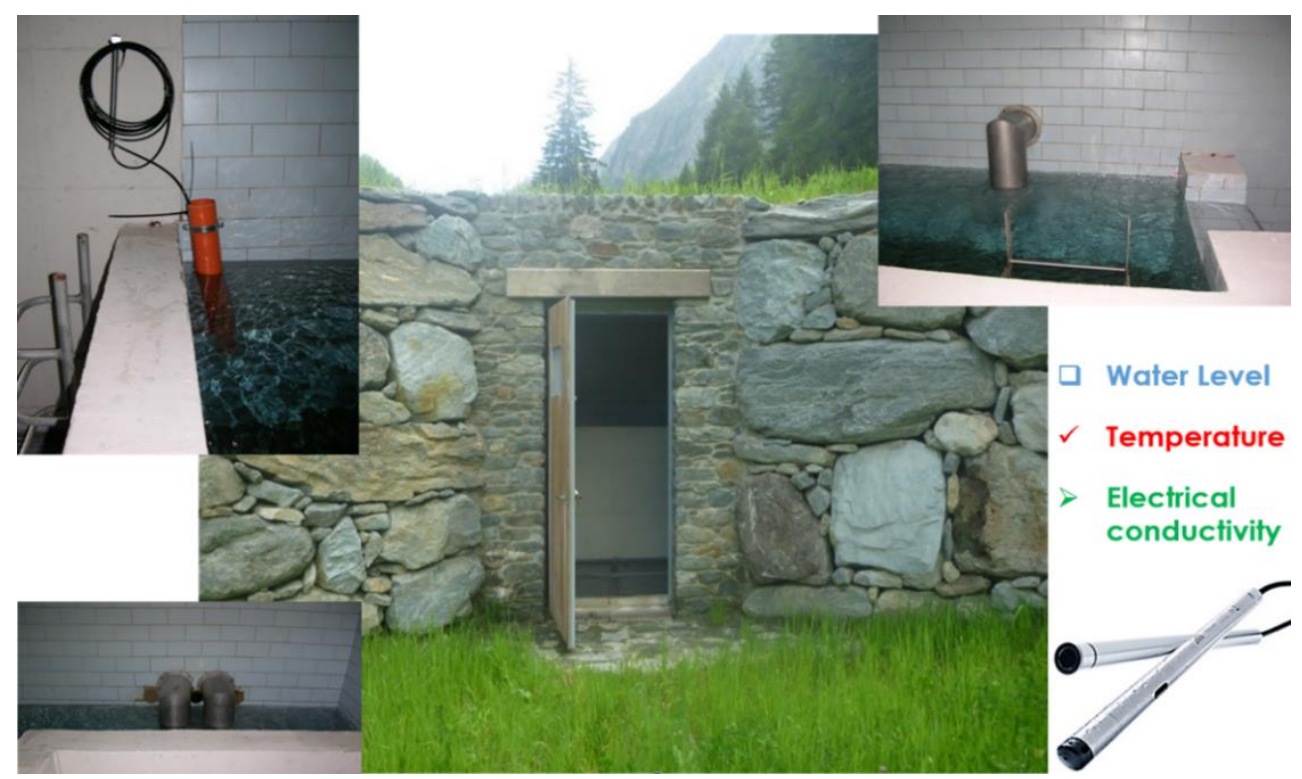

Figure 2: Overview of the instruments installed in the Mascognaz 1 spring

\subsection{Spring recession curve analysis}

118 Analysing spring discharge hydrographs is one of the most useful tools when studying mountain springs and

119 defining aquifer characteristics, such as the type and quantity of its groundwater reserves.

120 There have been several studies on recession curve modelling, each establishing different mathematical 121 relationships between the water spring discharge parameter $(Q)$ and recording time $(t)$. Boussinesq (1904) and 122 Maillet (1905) proposed two different analytical formulas that describe the dependence of the flow rate at a 123 specified time $\left(Q_{t}\right)$ on the flow rate at the beginning of the recession $\left(Q_{0}\right)$. These formulas allow for the 124 calculation of the available water volume at different moments in time. Boussinesq developed an exact analytical solution of the diffusion equation that describes flow through a porous medium by assuming a porous, free, homogeneous and isotropic aquifer limited by an impermeable horizontal layer at the level of the outlet:

$$
Q_{t}=\frac{Q_{0}}{\left(1+\alpha\left(t-t_{0}\right)^{2}\right.}
$$

128 where $Q_{t}\left(\mathrm{~m}^{3} / \mathrm{s}\right)$ is the flow rate value at $t \neq t_{0}, Q_{0}$ is the flow rate at $t=t_{0}$ and $\alpha$ is the recession coefficient, a constant that depends only on the aquifer hydraulic systems, as shown below. 


$$
\alpha=\frac{\sqrt{Q_{0}}-\sqrt{Q_{t}}}{\sqrt{Q_{t}} t}
$$

130 Maillet showed that the recession of a spring can be represented by an exponential formula, implying a linear

131 relationship between the hydraulic head and flow rate:

$$
Q_{t}=Q_{0} e^{-\alpha\left(t-t_{0}\right)}
$$

132 where the recession coefficient $\alpha$ can be determined using the following equation.

$$
\alpha=\frac{\log Q_{0}-\log Q_{t}}{e^{\left(t-t_{0}\right)}}
$$

133 In both methods, the recession coefficient equations are used to determine important hydrogeological

134 parameters: $W_{0}$, the groundwater volume stored above spring level at the end of the recharging season 135 (beginning of the recession; Eq. 5 and 7), and $W_{d}$, the groundwater volume stored at the end recession period 136 (Eq. 6 and 8).

Boussinesq (1904)

$$
\begin{gathered}
W o=\frac{Q_{0}}{\alpha(1+\alpha t)^{2}} \times 86400 \\
W_{d}=\left[\frac{Q_{0}}{\alpha}-\frac{Q_{0}}{\alpha(1+\alpha t)}\right] \times 86400
\end{gathered}
$$

Maillet (1905)

$$
\begin{gathered}
W_{0}=\frac{Q_{0}}{\alpha} \times 86400 \\
W_{d}=\frac{\left(Q_{0}-Q_{t}\right)}{\alpha} \times 86400
\end{gathered}
$$

\section{$137 \quad 2.3$ Autocorrelation and cross-correlation functions}

138 The autocorrelation function (ACF) can evaluate the linear dependency of successive values of a single 139 parameter for a defined time series. The method is univariate and quantifies the memory effect that corresponds 140 to the temporal reciprocal influence on subsequent data of a single dataset.

141 Statistically, the autocorrelation of a random process describes the correlation between values of the process 142 at different points in time as a function of the two times or the time difference. The autocorrelation for a 143 distance $\tau$ corresponds to the covariance of all measurements $x_{t}$ and measurements with a time distance $x_{\tau+\tau}$, 144 according to the following equation:

$$
\operatorname{cov} \tau=\frac{1}{n-\tau} \sum_{t=1+\tau}^{n} x_{t} x_{t-\tau}-X_{t} X_{t-\tau}
$$


where $x$ is a time series, $n$ is the number of measurements in the time series, $\tau$ is the time distance between two measurements, and $X$ is the average value of the sample. The autocorrelation coefficient (ACC) ranges from -1 to 1 . An ACC of 1 means that the compared time series are identical.

148 When using the ACF on hydrological data, a slow decline indicates an aquifer characterised by low draining 149 properties, low permeability or major groundwater storage. Conversely, a fast decline indicates a more rapid 150 flow of water through the aquifer and/or limited storage capacity (Imagawa et al. 2013; Reberski et al. 2013).

151 Traditionally, the ACC accounts for Q data, and the main hydrogeological assessment is obtained through such 152 an analysis. However, the ACC values can also be applied to T and EC, and these analyses can be used to 153 validate the hydrogeological view of the spring memory effect, which is based on Q data. The time-level 154 stabilities of T, EC and Q can be good markers of a high residence time in an aquifer (Lo Russo et al. 2015). 155 To identify any instances of pronounced similarity or linear correlation between individual data, two different 156 time series can be compared using the cross-correlation function (CCF; e.g., rainfall versus discharge 157 parameters). Cross-correlation analysis is based on an equation similar to the ACF. If two time series are 158 marked as variables $X$ and $Y$, and $n$ is the number of pairs that are compared in one step $(k)$ of the CCF, the 159 cross-correlation coefficient can be obtained by the following (Box and Jenkins 1974):

$$
R_{x y}(K)=\frac{n \sum X Y-\sum X \sum Y}{\sqrt{\left[n \sum X^{2}-\left(\sum X\right)^{2}\right] \times\left[n \sum Y^{2}-\left(\sum Y\right)^{2}\right]}}
$$

160 Values of $R_{x y}(K)$ can range between -1 (perfect negative correlation) and +1 (perfect positive correlation); a 161 value of 0 indicates no correlation.

162 As with the ACC, the CCF is an established technique that is usually applied on Q and P datasets. However, 163 the CCF can also assess T and EC datasets, and such analyses could be used to validate hydrogeological 164 considerations of the time lag response and maximum $R_{x y}(K)$ values.

165 Furthermore, the pollution vulnerability index of different springs can be estimated using the lag time derived 166 from cross-correlation analysis. This statistical method can be applied to explore the relation between discharge 167 and rainfall, as well as the relation between electrical conductivity and rainfall. 
Properly identifying the vulnerability level of a mountain aquifer and its associated springs is necessary to

170 protect aquifers from potential pollution sources and preserve water quality over time.

171 Aquifers are fed by rainfall, snowmelt and surface runoff, collectively known as neo-infiltration water. Water 172 from these sources infiltrates the ground and becomes part of the underground flow. Because neo-infiltration 173 water can transport pollutants into groundwater systems, the rate at which neo-infiltration water enters 174 groundwater systems and its velocity toward a spring must be accurately evaluated.

175 Galleani et al. (2011) combined monitored hydrogeological parameters and proposed a new analytical 176 approach called the VESPA (Vulnerability Estimator for Spring Protection Areas) index, which assesses the 177 behaviour of springs drainage systems through an analysis of their responses to different recharge impulses.

178 The VESPA index $V$ is defined using the following relationship (Banzato et al. 2017):

$$
V=c(\rho) \beta \gamma
$$

179 where $c(\rho)$ is the correlation factor. This factor is defined by the equation below.

$$
c(\rho)=[\mu(-\rho)+\alpha \mu(\rho)]|\rho|
$$

180 The $\rho$ value represents the correlation coefficient between Q and EC, calculated using over 1 year of continuous 181 hourly data. The function $\mu(\rho)$ is the Heaviside step function:

$$
u(\rho)=\{1 \rho \geq 0 ; 0 \rho<0\}
$$

182 where $\alpha$ is the scaling coefficient, which can range from 0 and 1 but is generally assumed to be 0.5 . The 183 variables $\beta$ and $\gamma$ are the temperature and discharge factors, respectively, and are defined by the following 184 equations:

$$
\begin{gathered}
\beta=\left\{\frac{T_{\text {max }}-T_{\min }}{1^{\circ} C}\right\}^{2} \\
\gamma=\frac{Q_{\text {max }}-Q_{\text {min }}}{Q_{\text {med }}}
\end{gathered}
$$

185 where $T_{\max }$ and $T_{\min }$ are the maximum and minimum temperatures, and $Q_{\max }, Q_{\min }$ and $Q_{\operatorname{med}}$ are the maximum, 186 minimum and average discharge values for the monitoring period.

187 According to Eq. (11), spring vulnerability is strictly related to a change in one of the parameters (Q, T or EC). 
189 infiltrative input. From the value of the correlation coefficient $\rho$, drainage systems can be classified into one 190 of three categories: highly effective (replacement effects prevail and $-1 \leq \rho \leq-0.2$ ); moderately effective 191 (piston flow prevails and $0.2 \leq \rho \leq 1$ ); and weakly effective (the homogenisation phenomenon prevails and $-0.2 \leq \rho \leq 0.2)$.

193 Based on the computed values of the VESPA index, a spring's vulnerability level can be defined according to 194 the classification in Table 1 (Galleani et al. 2011).

\begin{tabular}{|c|c|c|c|}
\hline \multicolumn{3}{|c|}{ VESPA Index Values and Their Vulnerability Levels } & \multirow{6}{*}{$\begin{array}{l}\text { Table 1: VESPA index values and their } \\
\text { vulnerability levels (Galleani et al. 2011) }\end{array}$} \\
\hline & & 195 & \\
\hline$V \geq 10$ & Very high & 196 & \\
\hline $1<V<10$ & High & 197 & \\
\hline $0.1<V<1$ & Medium & 198 & \\
\hline $0<V<0.1$ & Low & 199 & \\
\hline
\end{tabular}

\section{Code}

201 The dynamics of mountain groundwater resource depletion are heavily influenced by climate conditions. 202 Annual variations in snow and rain precipitation impact the hydrodynamic characteristics and exhaustion 203 modalities of springs. As such, it is necessary to develop new automated techniques that will allow researchers 204 to estimate the main parameters of mountain aquifers quickly and accurately. In order to propose a new, 205 advanced, semi-automatic tool for spring characterisation that uses available parameter datasets, the above206 described methodologies were implemented in Python.

207 Python is a high-level programming language with an object-oriented approach created by Guido van Rossum 208 in 1991. The first version of this study's Python code was developed in 2014 at Politecnico di Torino within 209 the framework of the Interreg Project between Italy and Switzerland. Over time, the tool was updated with the 210 latest libraries of Python. In order to improve software performance, the number of libraries used has been 211 limited, with preference given to common libraries over more experimental ones. In addition to the standard 212 Python libraries, code for the proposed tool utilized the following:

213 - Numpy (https://numpy.org/), the fundamental package for scientific computing; 
- Matplotlib (https://matplotlib.org/), a comprehensive library for creating static, animated and interactive visualisations; and

- Scipy (https://www.scipy.org/), a Python-based ecosystem of open-source software for mathematics, science and engineering.

218 The script of the final version of the code was connected to a Postgres Database, where all input information 219 is stored. The final program can accept tabulated data in an Excel spreadsheet and is usable in virtual 220 environments on Linux, Mac and Windows OS.

221 To be correctly input into SOURCE, Excel files must have a first sheet named "Spring_data" that contains the water spring data and a second sheet named "Meteo_data" that contains the meteorological data. With this format, it is possible to run the script and set parameters using the proposed GUI interface (Fig. 3). The 224 following information is also required:

- Filename: the file path of the Excel document, type string;

- Date start: the start date for the data, type string;

- Date stop: the stop date for the data, type string;

- Water spring name: name of the spring to be analysed, type string;

- Select type of analysis: a choice of 'All', 'Recession curves', 'VESPA vulnerability index', 'Plot data' or 'Auto \& Cross-correlation'; and

- Select method for recession curves: a choice of 'All', 'Maillet', 'Boussinesq' or 'none'.

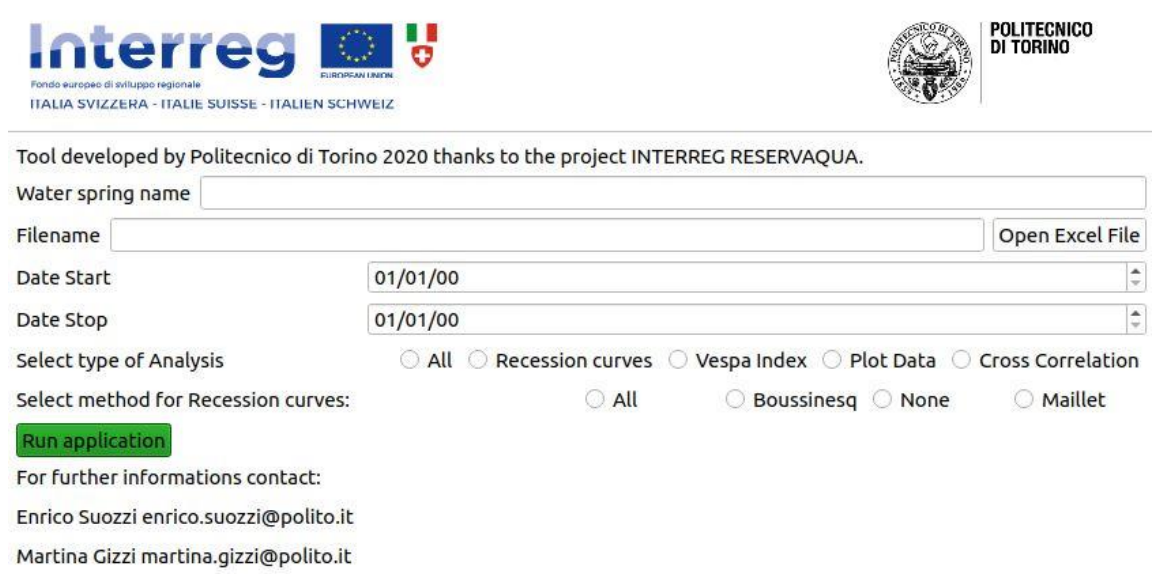

Figure 3: GUI of the proposed Python tool 


\section{Results}

233 Input data were recorded by the multiparametric probes installed in the Mascognaz 1 spring. Specific time 234 intervals were selected, and the data for this period were processed using the final version of the described 235 Python code. Graphical outputs and hydrodynamic parameter values for the analysed aquifer were obtained. 236 The monitoring datasets for the years 2012-2013, 2014-2015, 2015-2016 and 2016-2017 were selected for 237 analysis, and the outputs below were obtained and presented:

- Spring hydrographs

- Recession curves

- VESPA index

- Autocorrelation and cross-correlation coefficients

\subsection{Spring hydrograph}

243 The first graphical output that can be obtained are hydrographs for the spring under analysis. As reported in 244 Fig. 4, the Mascognaz 1 spring hydrographs were obtained by analysing data from the selected time range and 245 show variation in quick flow at the end of the winter period. Pronounced discharge fluctuations in the fast246 flow regime of the Mascognaz spring were due to contributions from snowmelt and the rapid infiltration of 247 precipitation during the autumn season. Abundant rainfall occurred during the autumn period of the selected 248 hydrographic years, causing the formation of a new peak and a decrease in the recorded values of T and EC. 249 Since the depletion curve was in approximately ideal conditions for each year analysed and was only weakly 250 influenced by infiltration events, the recession coefficients calculated using the Boussinesq (1904) and Maillet 251 (1905) methods can be considered reliable. 

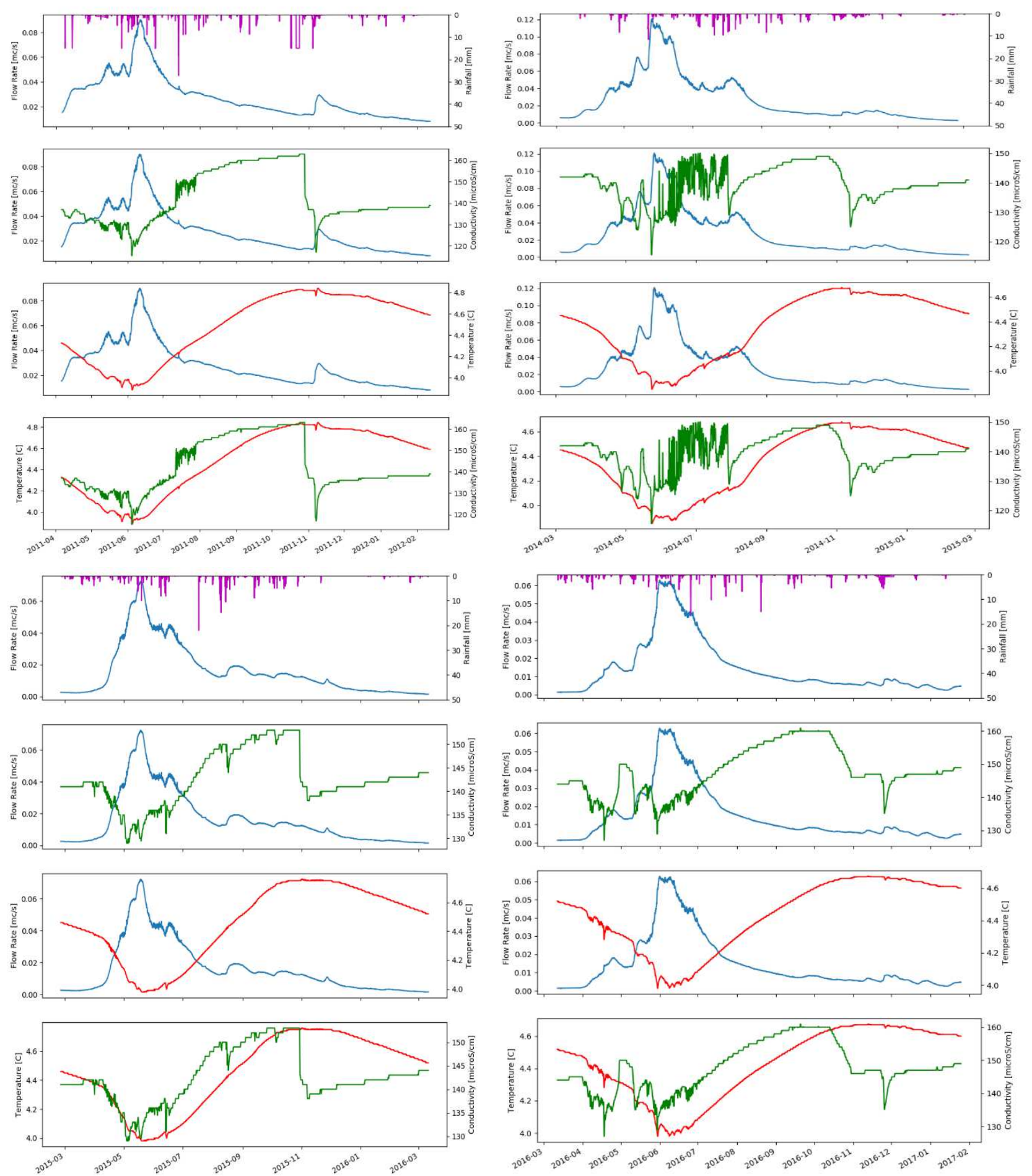

Figure 4: Mascognaz 1 spring hydrographs (NW Start 2011-04-06, Stop 2012-02-12; NE Start 2014-03-05 Stop 201502-24; SW Start 2015-02-25 Stop 2016-03-11; SE Start 2016-03-12 Stop 2017-01-25) for rainfall (violet lines), flow rate (blue lines), electrical conductivity (green lines) temperature (red lines)

\section{$255 \quad 4.2$ Recession curve}

256 The second possible graphical output is recession curves (Fig. 5). The aquifer parameters, calculated using the

Boussinesq (1904) and Maillet (1905) methods, are reported in Tab. 3. The flow rate estimates from the 
258 Boussinesq method closely matched the actual values reported for the years 2012-2013, 2015-2016 and 2017-

259 2018, but not for the year 2014-2015 (Fig. 5).

260 The duration of the exhaustion period, the time between snowmelt peaks and the annual minimum point before

261 the new recharge influence the $\alpha$ value. The minimum estimated value for $\alpha$ was 0.0094 (2011-2012, 262 Boussinesq) and the maximum value was 0.0206 (2014-2015, Boussinesq). The fluctuation depended mainly 263 on the amount of snow fall in winter (Tab. 2).

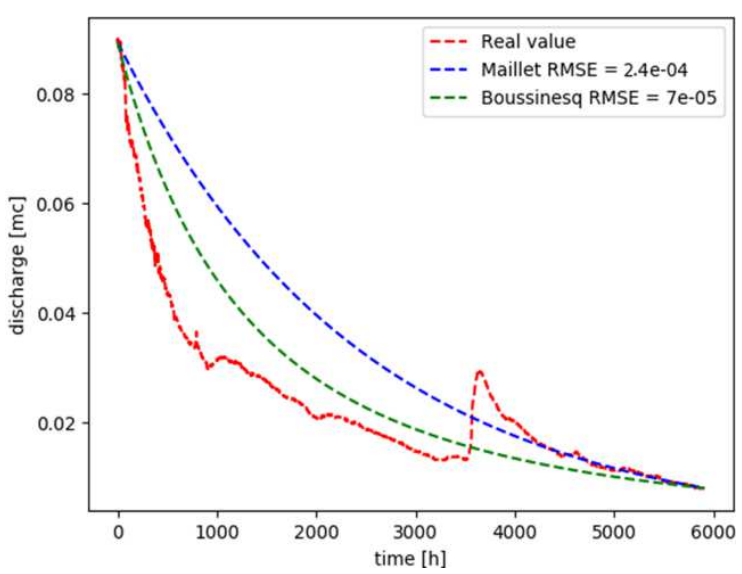

2011-2012

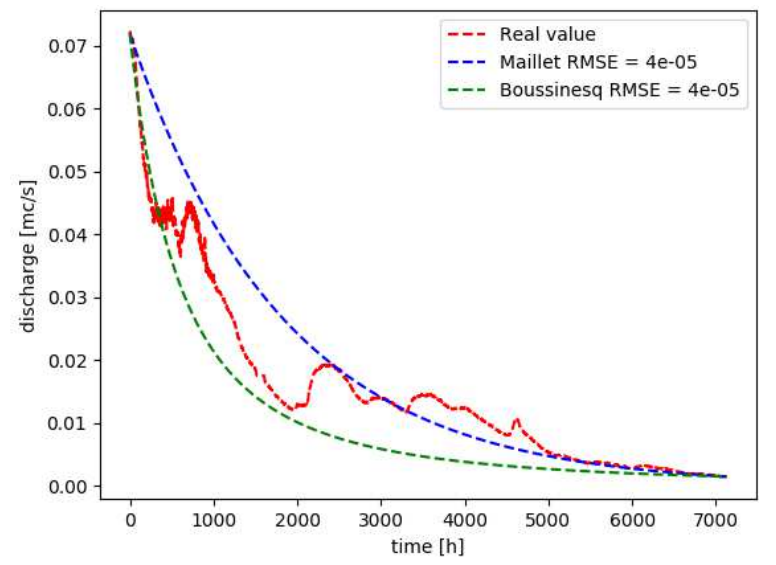

2015-2016

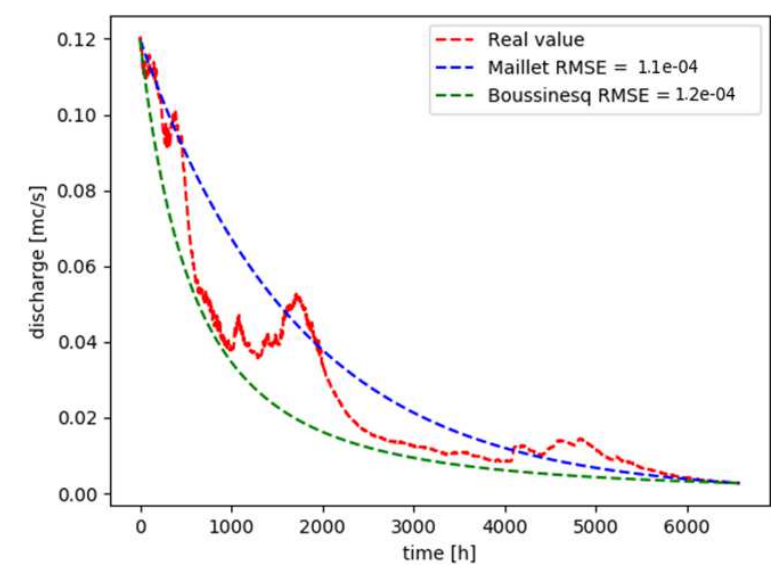

2014-2015

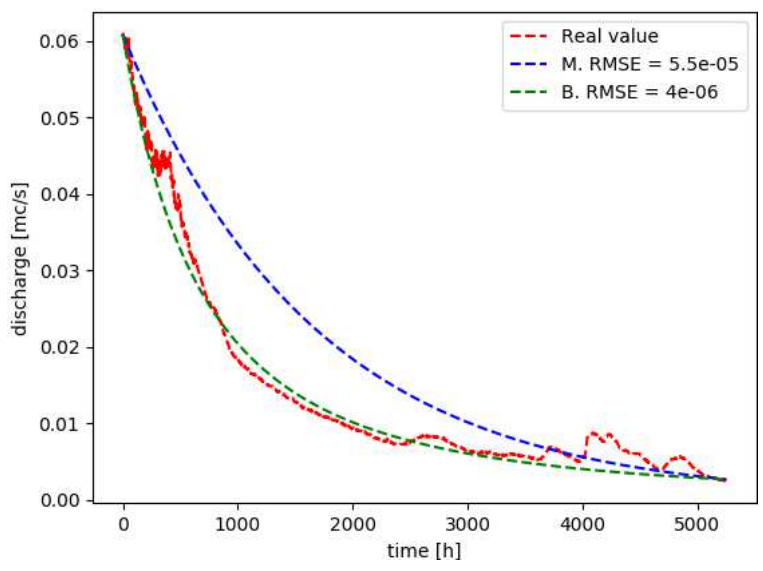

2017-2018 


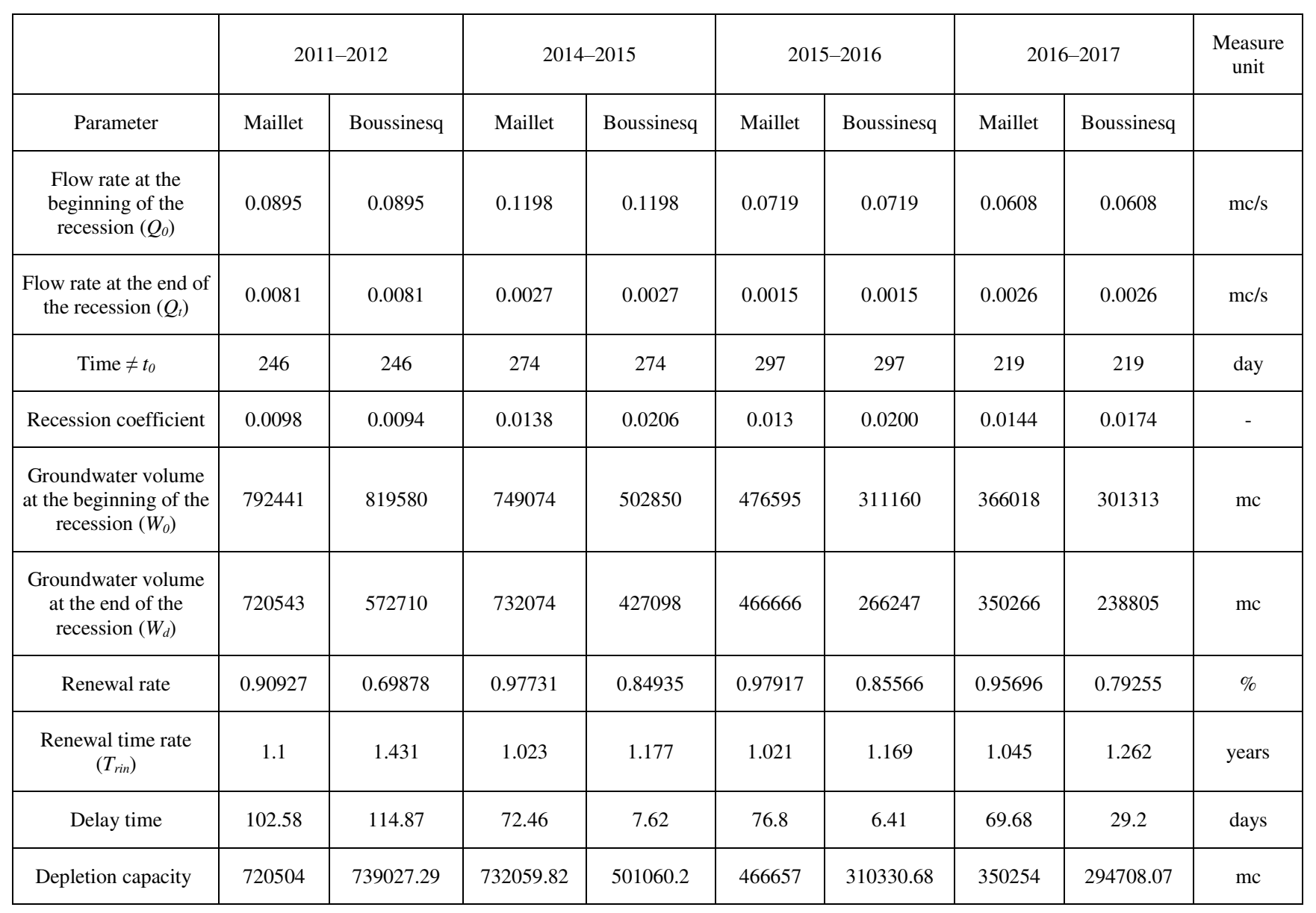

265 Table 2: Aquifer parameters calculated using the Boussinesq (1904) and Maillet (1905) methods

\section{$266 \quad 4.3$ The VESPA index}

267 The third type of information that can be obtained from SOURCE is the value of the VESPA index. The

268 vulnerability index of the spring had a medium value for all the years analysed (Tab. 3). The obtained $\rho$

269 correlation coefficients for each year in the selected timeframe identify the spring as a weakly effective

270 drainage system where the homogenisation phenomenon prevails.

\begin{tabular}{|c|c|c|c|c|}
\cline { 2 - 5 } \multicolumn{1}{c|}{} & $\mathbf{2 0 1 1}$ & $\mathbf{2 0 1 4}$ & $\mathbf{2 0 1 5}$ & $\mathbf{2 0 1 6}$ \\
\hline rho & 0.06 & 0.110 & 0.070 & 0.08 \\
\hline beta & 0.94 & 0.690 & 0.610 & 0.49 \\
\hline gamma & 3.08 & 5.040 & 3.970 & 3.91 \\
\hline VESPA index & 0.176 & 0.370 & 0.162 & 0.153 \\
\hline
\end{tabular}




\begin{tabular}{|l|l|l|l|l|}
\hline Vulnerability & Medium & Medium & Medium & Medium \\
\hline
\end{tabular}

271 Table 3: VESPA index values obtained for the Mascognaz 1 spring

$272 \quad 4.4$ Autocorrelation and cross-correlation coefficient

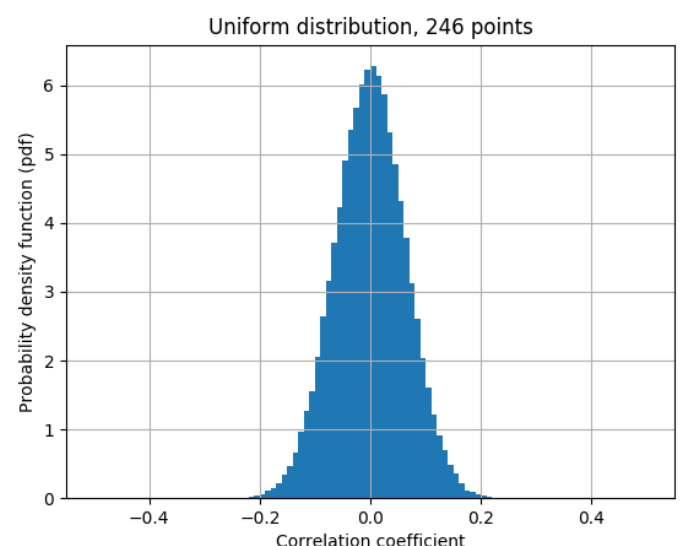

Figure 6: PDF of the estimated correlation coefficients (2011 dataset)

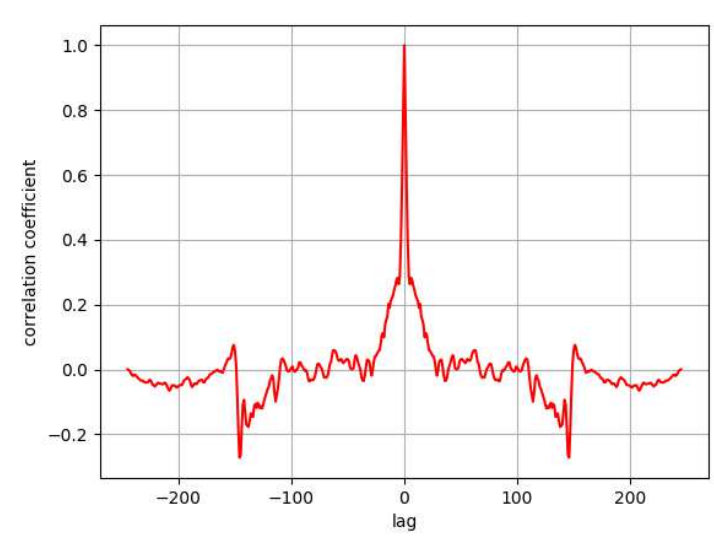

Figure 7: Estimated correlation coefficients, considering different time lags (2011 dataset)

273 The correlogram is a commonly used tool for checking the randomness of a dataset. If they are random,

274 autocorrelations should be near zero for any time-lag separations considered. The correlation analysis was first

275 performed on flow rate (Q) data. The distribution trend of the correlation coefficient reported in Fig. 6 can be

276 identified as a Gaussian curve; the correlation coefficient values are concentrated in a narrow range of values,

277 and so the maximum autocorrelation is obtained with a very short lag time (Fig.7).

\begin{tabular}{|l|l|l|l|l|}
\hline & 2011 & 2014 & 2015 & 2016 \\
\hline Correlation coefficient & 0.107 & 0.2 & 0.15 & 0.11 \\
\hline
\end{tabular}

278 Table 4: Correlation coefficient values obtained for the Mascognaz spring 1, considering different analysed datasets

279 As reported in Tab. 4, the correlation values tended to change over time. The Mascognaz springs 280 autocorrelation lag varied within a narrow band across years, implying the series is not significantly correlated 
281 with the delayed series; the variations from one instant or period to another are random phenomena (i.e., there 282 is an accidental component, or the stochastic part prevails; Fig. 8).
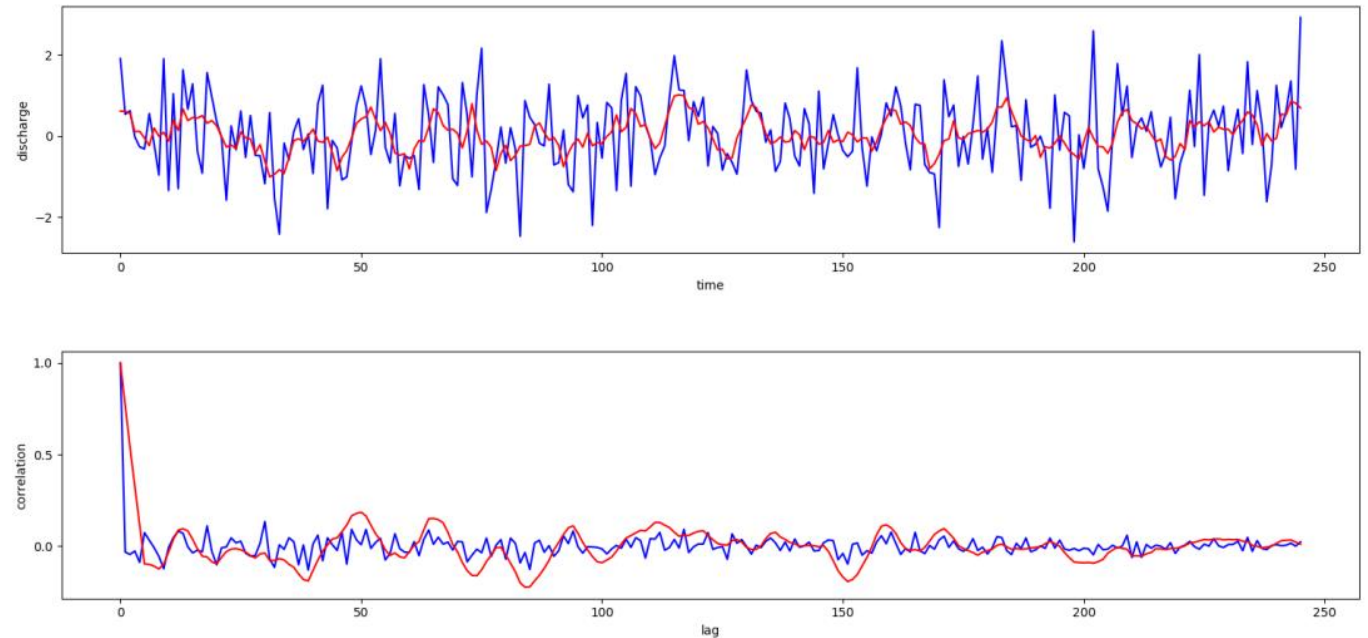

Figure 8: Autocorrelation diagrams

283 Understanding the correlation period between precipitation and spring response requires eliminating the 284 influence of the winter recharge period until the peak due to melting. Springs do not respond to winter and 285 spring precipitation; the water that arrives at springs depends on the melting process, which is correlated with 286 temperature fluctuations rather than precipitation.

287 Precipitation values were recorded by the Mascognaz meteorological station. The type of precipitation (solid 288 or liquid) was determined using the Parsivel 2 of the OTT, a modern laser disdrometer. The beginning of the 289 period selected for cross-correlation analysis was considered coincident with the beginning of the exhaustion period; this was also true for the recession analysis using the Maillet and Boussinesq methods. 
291 Comparing data from different years within the selected period revealed that the lag time between rainfall and 292 discharge tended to be short. As shown in Fig. 9, the considered lag time was 3 days; analysing all years within 293 the considered period resulted in a maximum value of 4 days and a minimum value of 1 (Tab. 5).
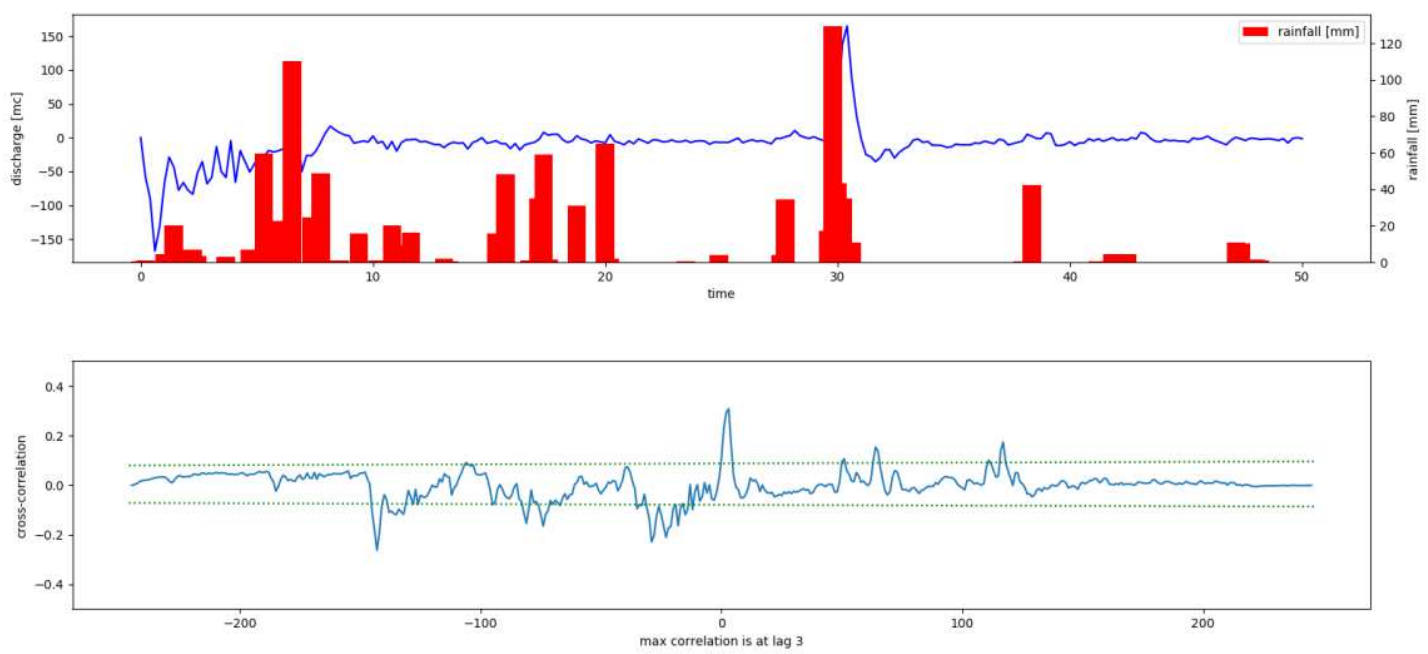

Figure 9: Cross correlation between rainfall and flow rate

\begin{tabular}{|c|c|c|c|c|}
\hline Year & 2011 & 2014 & 2015 & 2016 \\
\hline $\begin{array}{c}\text { Cross correlation } \\
\text { (lag time) }\end{array}$ & 3 & 1 & 1 & 2 \\
\hline
\end{tabular}

Table 5: Cross-correlation coefficient values obtained for the Mascognaz 1 spring

295 To verify the validity of the tool proposed and presented in this work, all of the results derived from the application were checked with manual calculations carried out by using Excel spreadsheets. By comparing the values obtained for the hydrodynamic parameters of the aquifer $\left(\alpha, W_{0}\right.$ and $\left.W_{d}\right)$ and the estimated indexes of vulnerability ( $V$ index) for all studied seasons, the proposed tool was found to be completely reliable.

\section{Conclusions}

300 New automated tools can potentially be applied to estimate aquifer hydrogeological parameters and monitor 301 water spring behaviour. The effects of climate change on mountain springs can be intense, and tools are needed 302 to guarantee a correct understanding of the dynamics of available resource exhaustion.

303 In this paper, SOURCE is an advanced semi-automatic Python tool that automates the hydrogeological 304 characterisation of spring aquifers. This tool was tested through an analysis of the Mascognaz 1 mountain 
springs. Graphical outputs, as well as hydrodynamic parameter values (e.g., VESPA index and auto- and crosscorrelation coefficients) for an aquifer, can be obtained from SOURCE. These graphs and values are crucial

307 for understanding the hydrogeological processes that characterise spring aquifers and for developing a proper 308 groundwater resource management strategy.

309 Unlike the software currently available through various university centres (e.g., RC software and the USGS

310 GW Toolbox), the proposed tool provides an accurate estimation of the vulnerability index and also provides

311 a recorded signal analysis using autocorrelation and cross-correlation statistical functions. A single software

312 package that contains all of the main methods of water spring analysis has the potential to significantly reduce 313 analysis times.

314 The SOURCE intuitive interface allowed not only researchers and hydrogeologists, but also non-expert users

315 to test the software and correctly use its functionalities for mountain springs analysis.

316 SOURCE is an open-source software tool, and the code is available for free download at 317 https://www.diati.polito.it/ricerca/aree/geologia_applicata_geografia_fisica_e_geomorfologia.

318 The authors are open to all comments and advice from users that could help to further implement the code and 319 improve the performances.

\section{Declarations}

$321 \quad$ Funding

322 Not applicable

323 Conflicts of interest

324 The authors declare that they have no known competing financial interests or personal relationships that 325 could have appeared to influence the work reported in this paper.

\section{Availability of data and material}

327 The hourly recorded data used to support the findings of this study have not been made directly available 328 because they are ownership of Politecnico di Torino. However, they are reported as graphs.

\section{Code availability}

330 The code is available for free download at

331 https://www.diati.polito.it/ricerca/aree/geologia applicata geografia fisica e geomorfologia 
Aller L, Bennet T, Lehr J H, Petty R J, Hackett G (1987). "DRASTIC: A Standardized System for Evaluating Environmental Protection Agency.

Amit H, Lyakhovsky V, Katz A, Starinsky A, Burg A (2002). Interpretation of spring recession curves. Ground Water 40(5):543-551.

Angelini P (1997). Correlation and spectral analysis of two hydrogeological systems in Central Italy. Hydrol. Sci. J. 42, 425-438. https://doi.org/10.1080/02626669709492038

Amanzio G, De Maio M, Suozzi E, Bertolo D, Lodi L P, Pitet L (2015). Global Warming in the Alps: Vulnerability and Climatic Dependency of Alpine Springs in Italy. Regione Valle d'Aosta and Switzerland, Canton Valais; G. Lollino et al. (eds.). Engineering Geology for Society and Territory Volume 5, DOI: 10.1007/978-3-319-09048-1_263. Springer International Publishing Switzerland 2015

Amanzio G, De Maio M, Suozzi E (2015). Vulnerability of Mountain Springs Affect by Climatic Change: A New Method in a Porous Media Aquifer in Regione Automa Valle d'Aosta G. Lollino et al. (eds.). Engineering Geology for Society and Territory - Volume 5, DOI: 10.1007/978-3-319-09048-1_259. Springer International Publishing Switzerland 2015

Banzato C, Butera I, Revelli R, Vigna B (2017). Reliability of the VESPA index in identifying spring vulnerability level. J. Hydrol. Eng. 22, 1-11. https://doi.org/10.1061/(ASCE)HE.1943-5584.0001498

Barlow P M, Cunningham W L, Zhai T and Gray M (2017). U.S. Geological Survey Groundwater Toolbox version 1.3.1, a graphical and mapping interface for analysis of hydrologic data: U.S. Geological Survey Software Release, 26 May 2017, http://dx.doi.org/10.5066/F7R78C9G

Boussinesq J (1904). Recherches théoriques sur l'écoulement des nappes d'eau infiltrées dans le sol et sur le débit des sources. J. Math. Pure Appl. 10:5-78

Cambi C, Dragoni W (2000). Groundwater yield, climatic changes and recharge variability: considerations arising from the modelling of a spring in the Umbria-Marche Apennines. Hydrogéologie, n.4, ed. BRGM, pp. 11-25.

Christe P, Amanzio G, Suozzi E, Mignot E, Ornstein P (2013). Global warming in the Alps: vulnerability and climatic dependency of alpine springs in Italy, Regione Valle d'Aosta (Italy) and Canton Valais (Switzerland). Eur Geol-J Eur Fed Geol 35:64-69 (ISSN:1028-267X)

Civita M, De Maio M (1997). Sintacs. Un sistema parametrico per la valutazione e la cartografia della vulnerabilità degli acquiferi all'inquinamento. Metodologia e automatizzazione, Manuali di protezione delle acque sotterranee. Pitagora

Dal Piaz G V (1992). Alpi dal Monte Bianco al Lago Maggiore. Guide geologiche regionali. Soc. Geol. It. BEMA ed., 3(2)

Dewandel B, Lachassagne P, Bakalowicz M, Weng P, Al-Malki A (2003). Evaluation of aquifer thickness by analysing recession hydrographs. Application to the Oman ophiolite hard-rock aquifer. J. Hydrol. 274, 
Galleani L, Vigna B, Banzato C, Lo Russo S (2011). Validation of a Vulnerability Estimator for Spring Protection Areas: The VESPA index. J. Hydrol. 396, 233-245. https://doi.org/10.1016/j.jhydrol.2010.11.012

Gattinoni P, Francani V (2010). Depletion Risk Assessment of the Nossana Spring (Bergamo, Italy) Based on the Stochastic Modeling of Recharge. Hydrogeol. J. 18, 325-337. https://doi.org/10.1007/s10040-009$\underline{0530-3}$

Gizzi M, Lo Russo S, Forno M G, Cerino Abdin E, Taddia G (2020). Geological and Hydrogeological Characterization of Springs in a DSGSD Context (Rodoretto Valley - NW Italian Alps). Appl. Geol. 319. https://doi.org/10.1007/978-3-030-43953-8_1

Gogu R, Dassargues A (2000). Current trends and future challenges in groundwater vulnerability assessment using overlay and index methods. Environ. Geol. 39, 549-559. https://doi.org/10.1007/s002540050466

Imagawa C, Takeuchi J, Kawachi T, Chono S, Ishida K (2013). Statistical analyses and modeling approaches tohydrodynamic characteristics in alluvial aquifer.Hydrol. Process., 27, 4017-4027

Kova'cs A, Perrochet P, Kira'ly L, Jeannin P Y (2005). Aquantitative method for the characterization of karst aquifersbased on spring hydrograph analysis. Journal of Hydrology 303, 152-164

Larocque M, Mangin A, Razack M, Banton O (1998). Contribution of correlation and spectral analyses to the regional study of a large karst aquifer (Charente, France). J. Hydrol. 205, 217-231. https://doi.org/https://doi.org/10.1016/S0022-1694(97)00155-8

Lo Russo S, Amanzio G, Ghione R, De Maio M (2015). Recession hydrographs and time series analysis of springs monitoring data: application on porous and shallow aquifers in mountain areas (Aosta Valley). Environ. Earth Sci. 73, 7415-7434. https://doi.org/10.1007/s12665-014-3916-Z

Maillet E (1905). Essais d'hydraulique souterraine et fluviale, Vol. 1. Herman et Cie, Paris, pp 218. 
J, Wilke K (2001). Impact of Climate Change on Hydrological Regimes and Water Resources

Panagopoulos G, Lambrakis N (2006). The contribution of time series analysis to the study of the hydrodynamic characteristics of the karst systems: Application on two typical karst aquifers of Greece (Trifilia, Almyros Crete). J. Hydrol. - J HYDROL 329, 368-376. https://doi.org/10.1016/j.jhydrol.2006.02.023

Reberski Lukac` J, Markovic' T, Nakic` Z (2013). Definition of the River Gacka springs subcatchment areas on the basis of hydrogeological parameters. Geologia Croatica 66(1):39-53. doi:10.4154/gc. 2013.04

Tague C, Choate J, Grant G (2012). Parameterizing sub-surface drainage with geology to improve modeling streamflow responses to climate in data limited environments. Hydrol. Earth Syst. Sci. Discuss. 9. https://doi.org/10.5194/hessd-9-8665-2012 


\section{Figures}

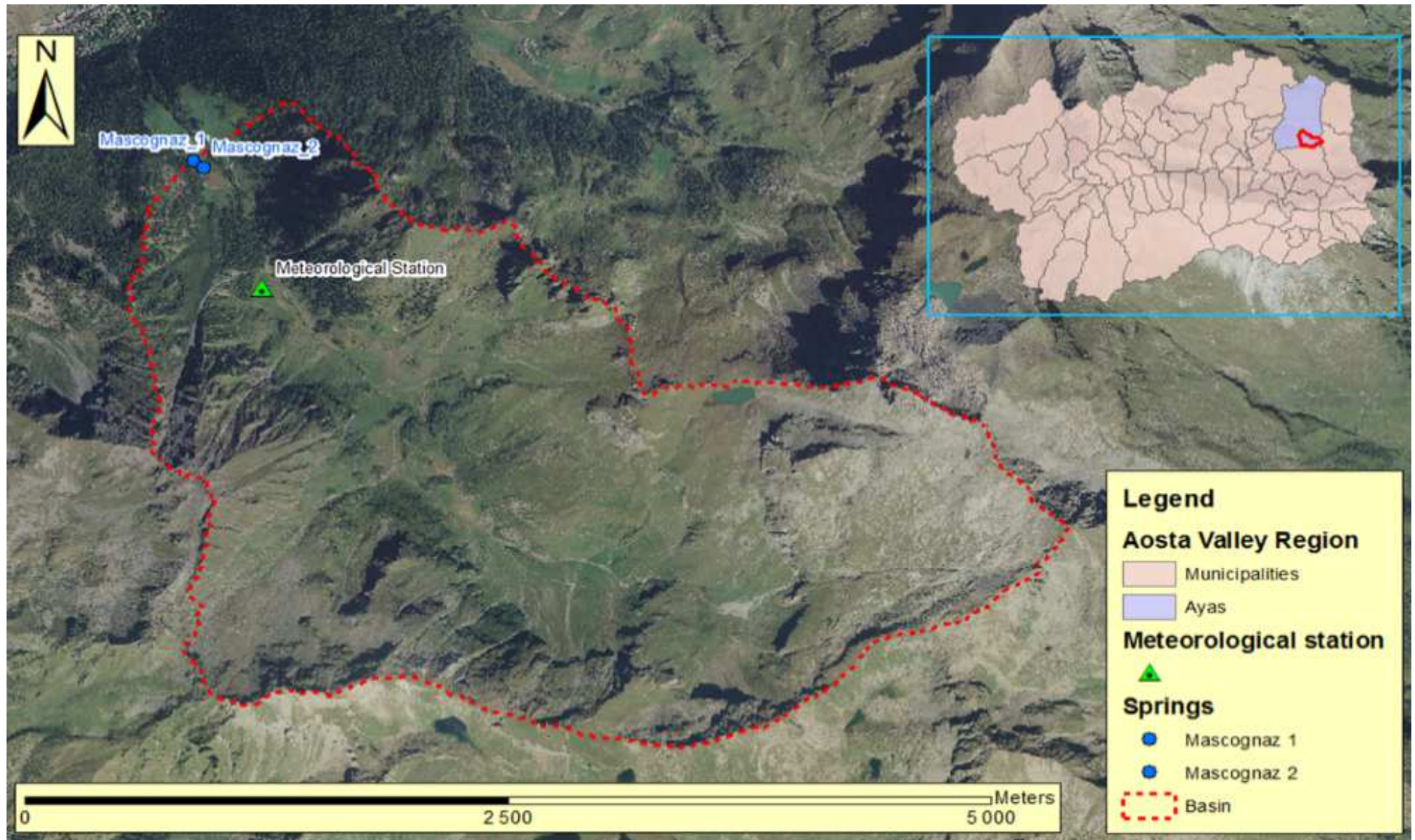

\section{Figure 1}

Mascognaz springs location Note: The designations employed and the presentation of the material on this map do not imply the expression of any opinion whatsoever on the part of Research Square concerning the legal status of any country, territory, city or area or of its authorities, or concerning the delimitation of its frontiers or boundaries. This map has been provided by the authors. 


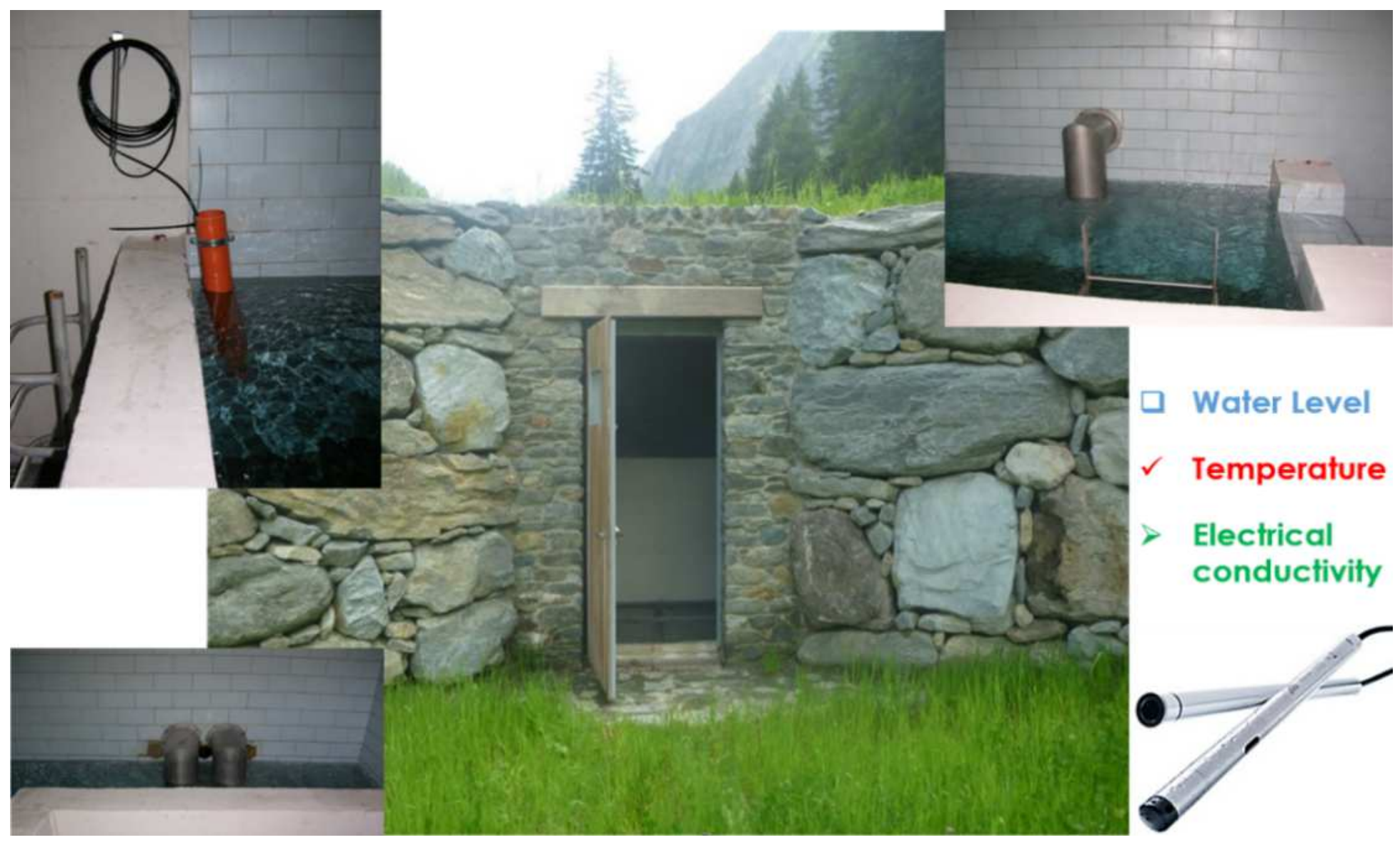

Figure 2

Overview of the instruments installed in the Mascognaz 1 spring

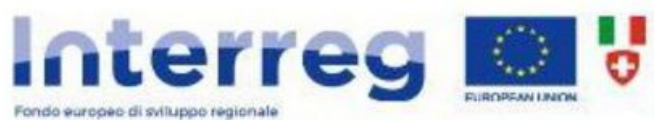

IIALIA SVIZLERA - ITALIE SUISSE - IIALIEN SCHWEIZ

POLITECNICO

Tool developed by Politecnico di Torino 2020 thanks to the project INTERREG RESERVAQUA.

Water spring name

Filename

Open Excel File

Date Start

$01 / 01 / 00$

Date Stop

$01 / 01 / 00$

Select type of Analysis

All Recession curves Vespa Index Plot Data cross Correlation

Select method for Recession curves:

All

Boussinesq None

Maillet

Run application

For further informations contact:

Enrico Suozzi enrico.suozzi@polito.it

Martina Gizzi martina.gizzi@polito.it 
GUI of the proposed Python tool
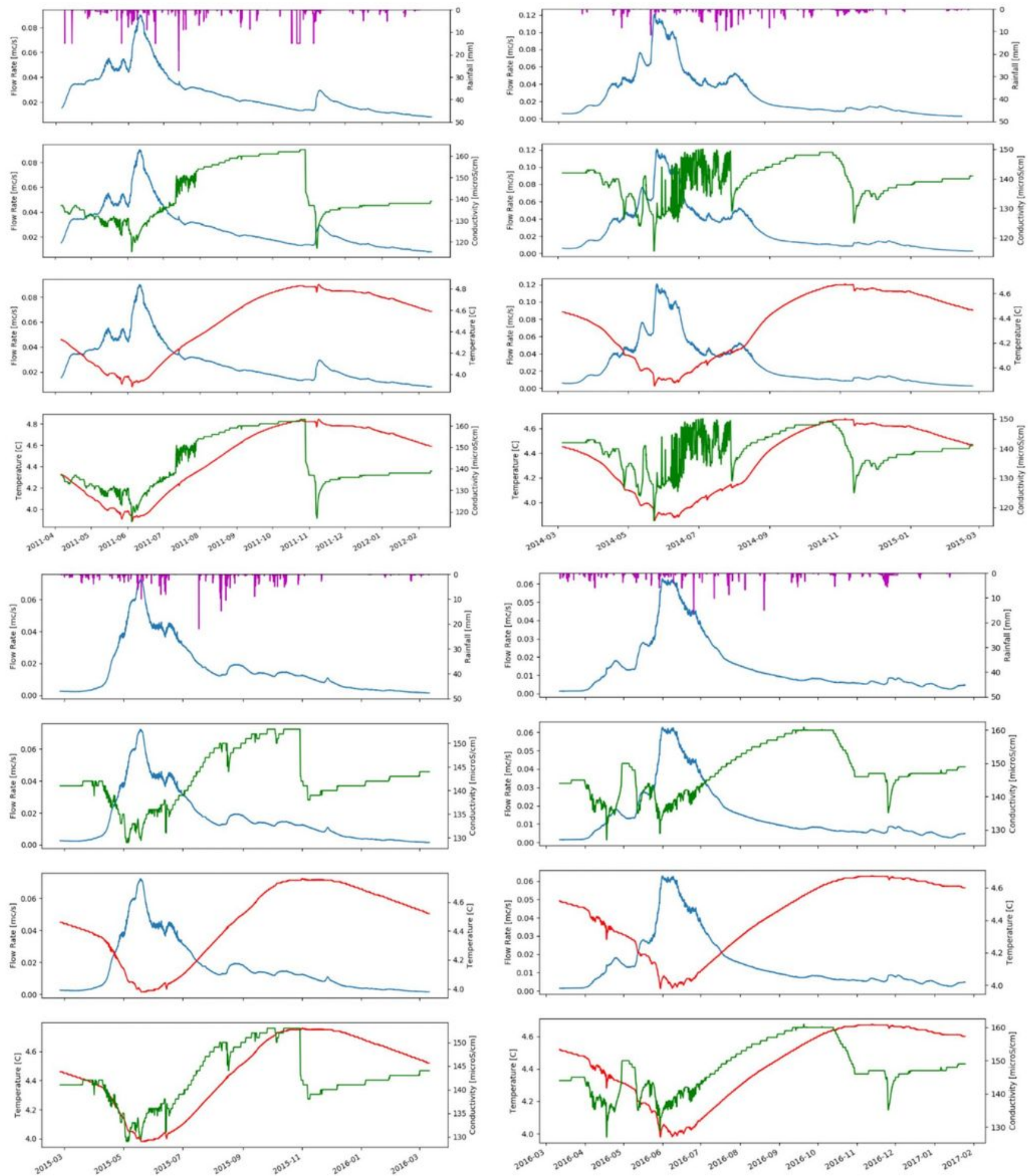

\section{Figure 4}

Mascognaz 1 spring hydrographs (NW Start 2011-04-06, Stop 2012-02-12; NE Start 2014-03-05 Stop 2015-02-24; SW Start 2015-02-25 Stop 2016-03-11; SE Start 2016-03-12 Stop 2017-01-25) for rainfall (violet lines), flow rate (blue lines), electrical conductivity (green lines) temperature (red lines) 


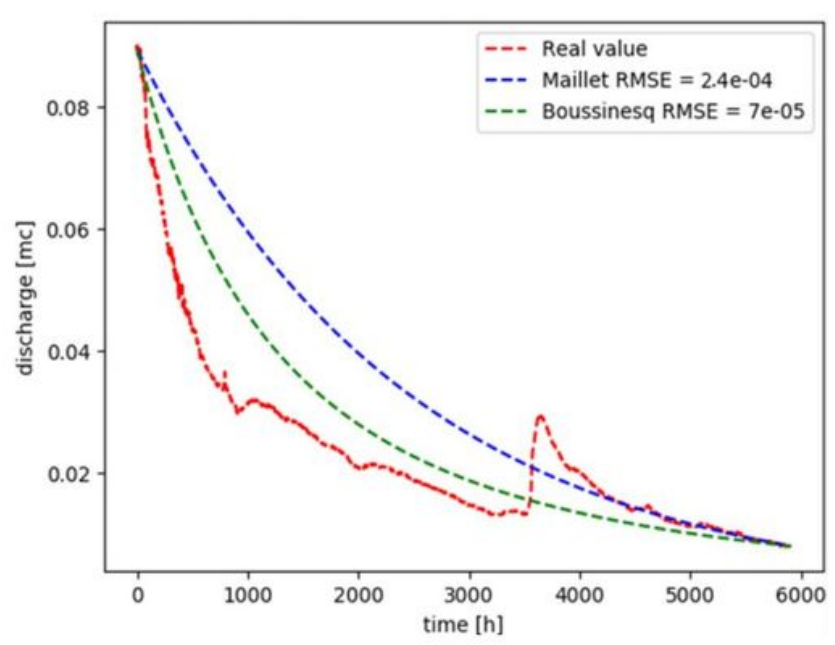

2011-2012

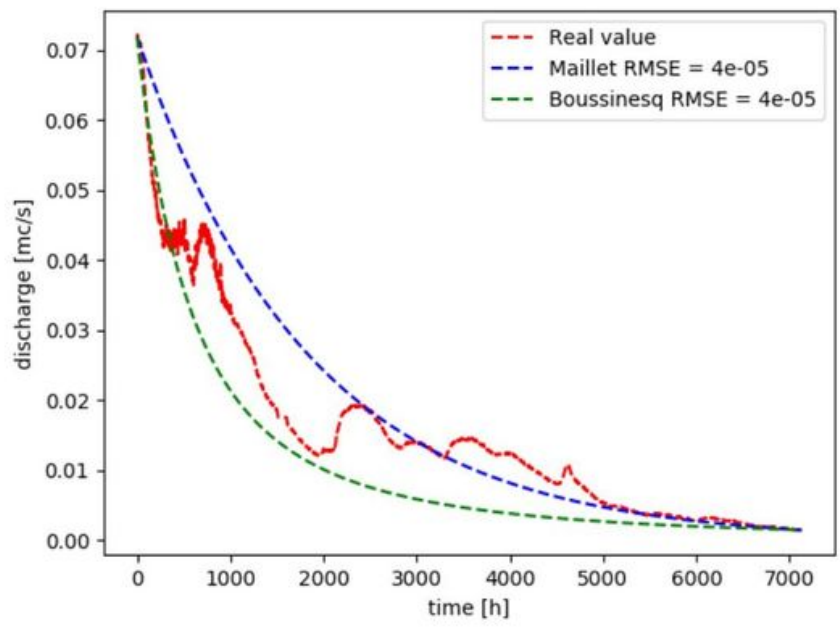

2015-2016

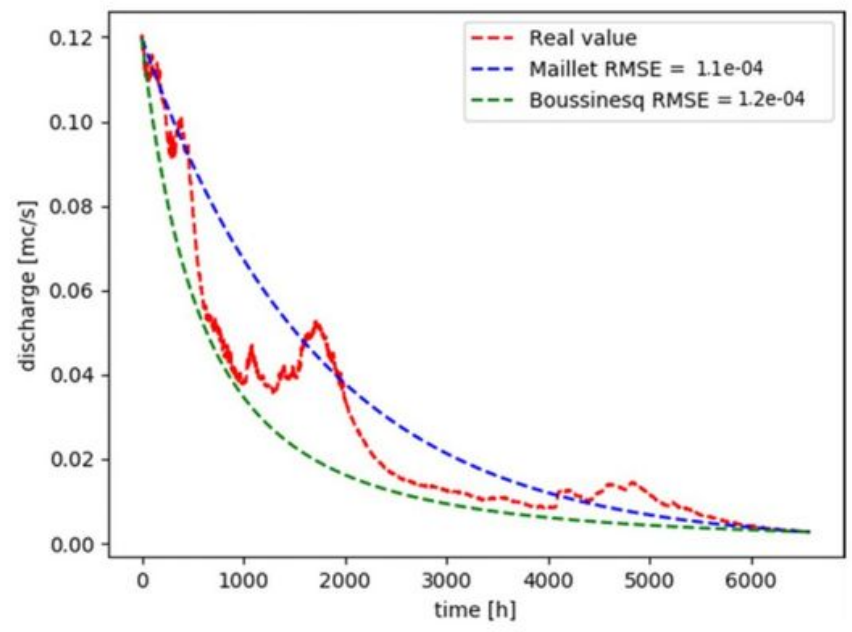

2014-2015

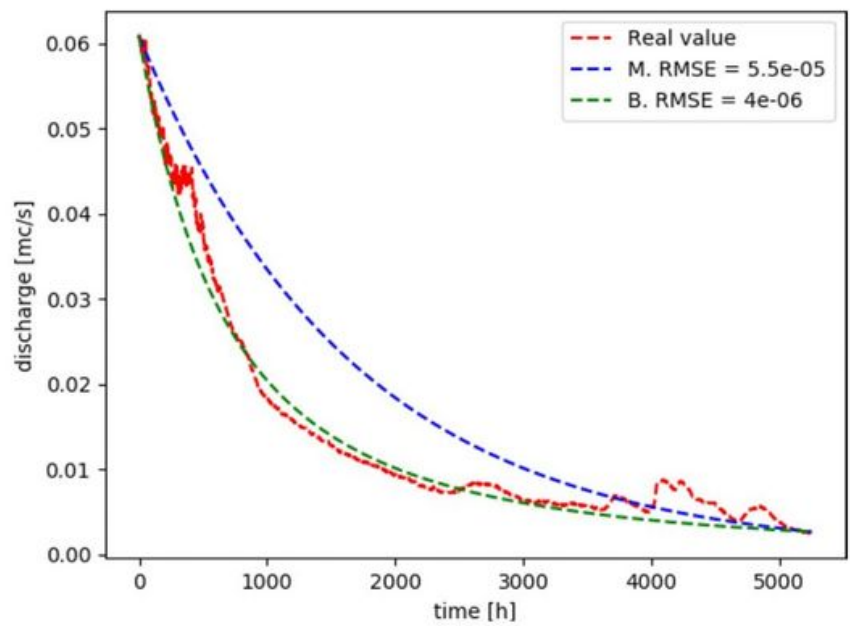

$2017-2018$

Figure 5

Mascognaz 1 recession curves based on the Boussinesq (1904) and Maillet (1905) methods 


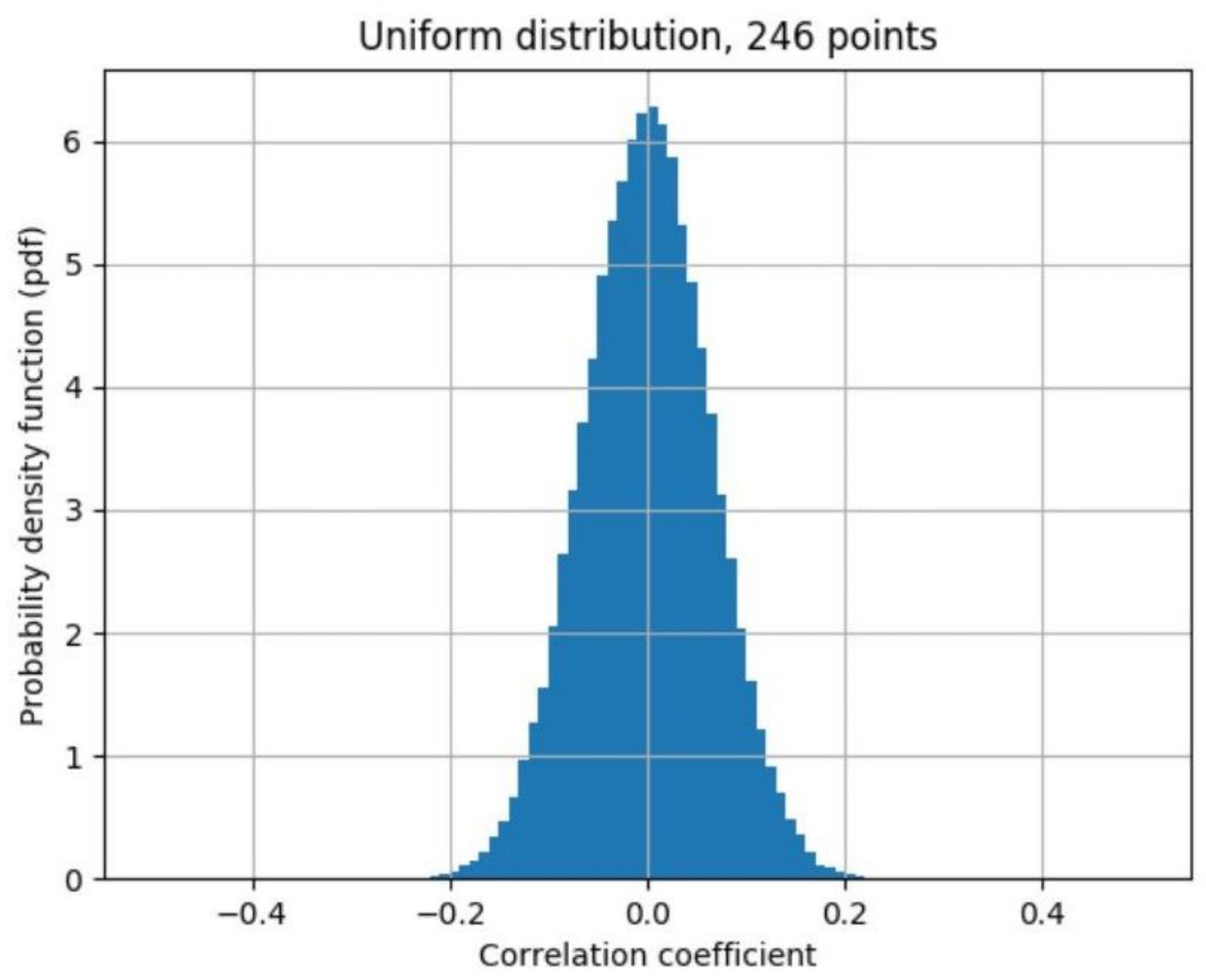

Figure 6

PDF of the estimated correlation coefficients (2011 dataset) 


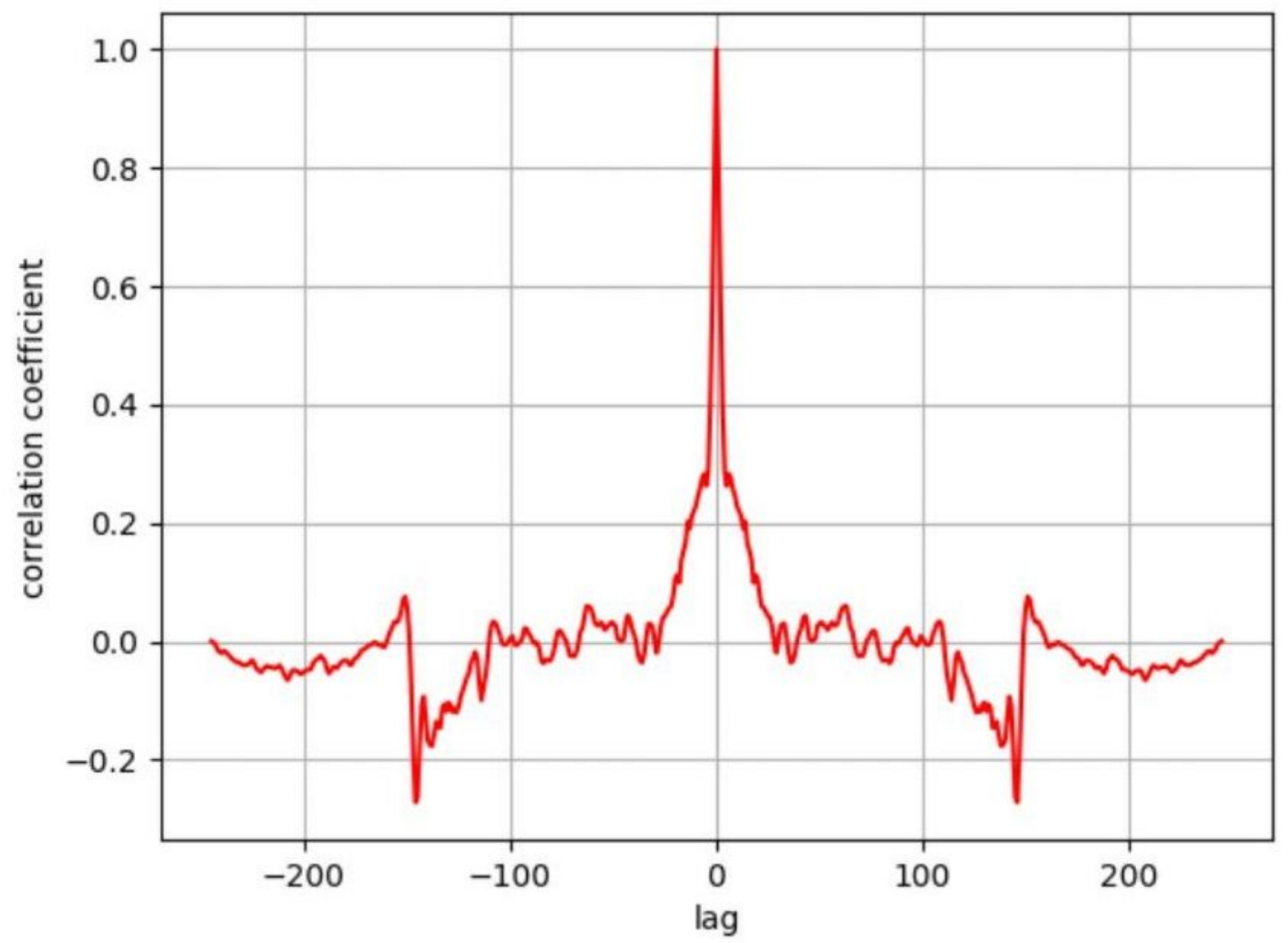

Figure 7

Estimated correlation coefficients, considering different time lags (2011 dataset) 

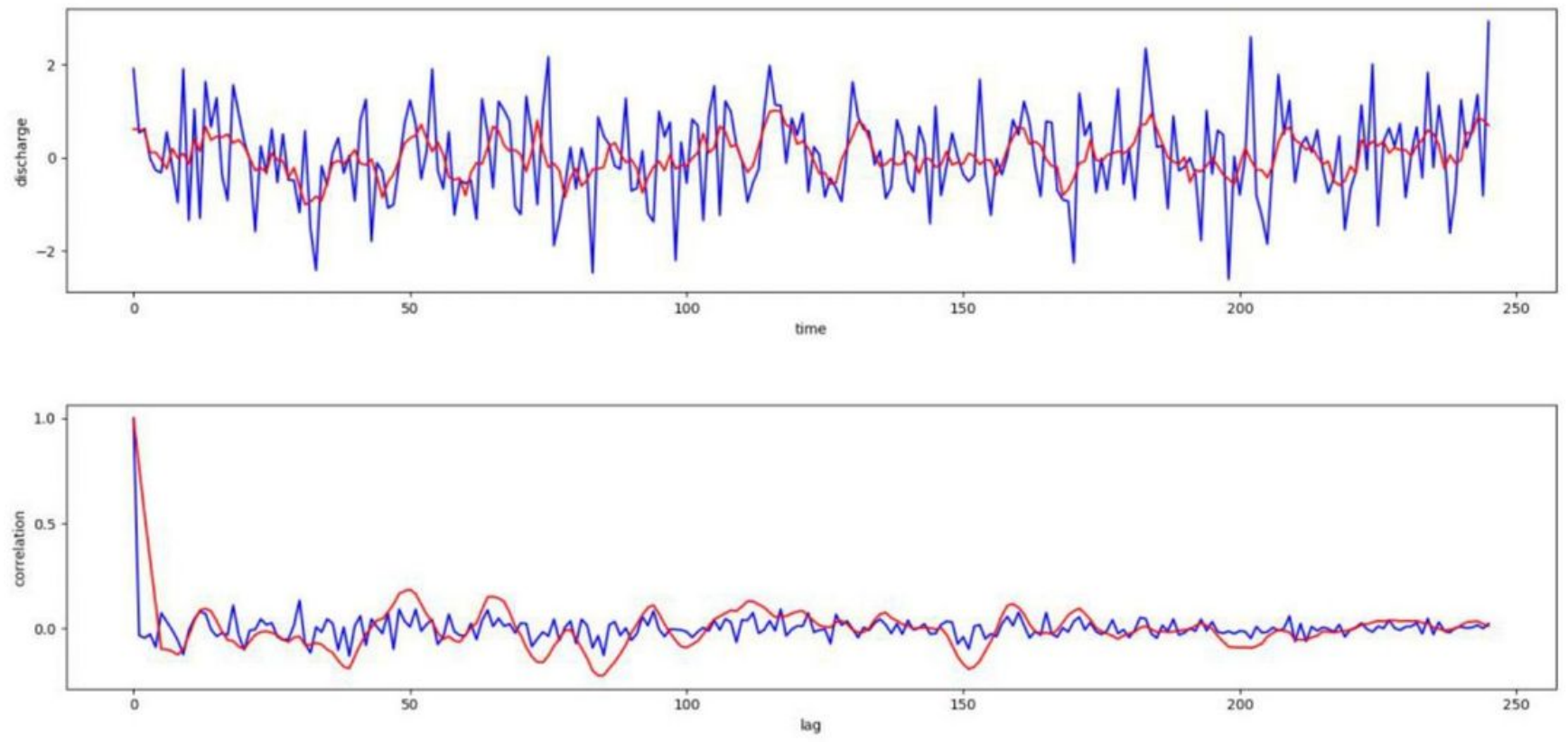

Figure 8

Autocorrelation diagrams
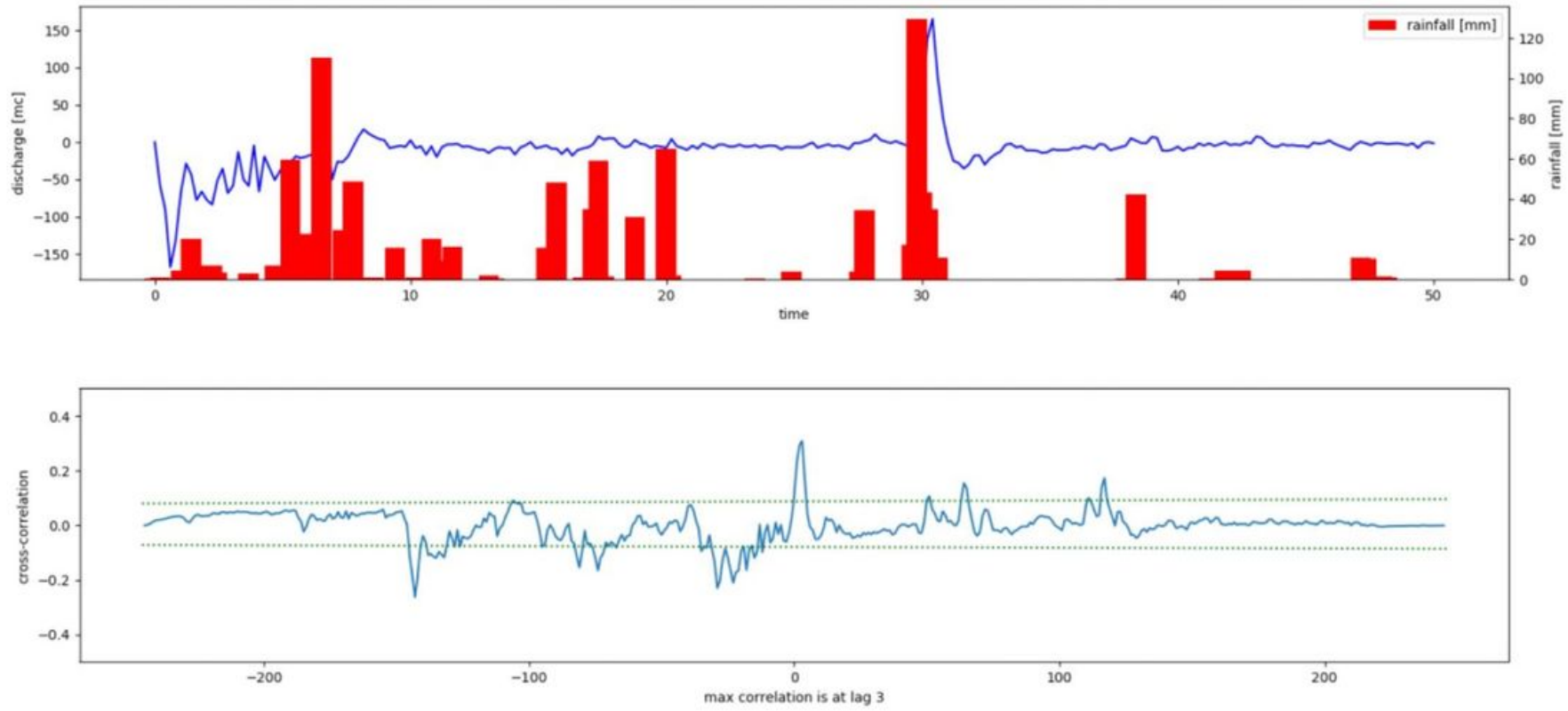

Figure 9

Cross correlation between rainfall and flow rate 